\title{
Electromechanical Dyssynchrony and Clinically Silent Ventricular Dysfunction in Young Subjects with Ventricular Pacing for Congenital and Early Acquired Av Block
}

\author{
Daniel Forsha ${ }^{1,9 *}$, David G Gamboa ${ }^{2,9}$, Niels Risum ${ }^{3}$, P Andrea Kropf ${ }^{4}$, Christoph P Hornik ${ }^{5}$, Piers \\ Barker $^{6}$, Joseph Kisslo ${ }^{7}$ and Ronald J Kanter ${ }^{8}$ \\ ${ }^{1}$ Ward Family Heart Center, Children's Mercy Kansas City, Kansas City, MO, USA \\ ${ }^{2}$ Division of Pediatric Cardiology, Advocate Children's Hospital, Oak Lawn, IL, USA
}

${ }^{3}$ Department of Cardiology, Hvidovre University, Hvidovre, Denmark

${ }^{4}$ Division of Pediatric Cardiology, University of Florida School of Medicine, Jacksonville, FL, USA

${ }^{5}$ Duke Clinical Research Institute, Duke University Medical Center, Durham, NC, USA

${ }^{6}$ Division of Pediatric Cardiology, Duke University Medical Center, Durham, NC, USA

${ }^{7}$ Division of Cardiovascular Disease, Duke University Medical Center, Durham, NC, USA

${ }^{8}$ Division of Cardiology, Miami Children's Hospital, Miami, FL, USA

${ }^{9}$ These authors contributed equally to this work

*Corresponding author: Daniel Forsha, Ward Family Heart Center, Children’s Mercy Kansas City, Kansas City, MO, USA

\section{ARTICLE INFO}

Received: 幽 July 25, 2019

Published: 㗀 August 06, 2019

Citation: Daniel Forsha, David G Gamboa, Niels Risum, P Andrea Kropf, Christoph P Hornik, et al. Electromechanical Dyssynchrony and Clinically Silent Ventricular Dysfunction in Young Subjects with Ventricular Pacing for Congenital and Early Acquired Av Block. Biomed J Sci \& Tech Res 20(2)-2019. BJSTR. MS.ID.003435.

Keywords: Electromechanical Delay; Strain; Cardiac Pacing, LV: Left Ventricular

Abbreviations: AV: Complete Atrioventricular, RVP: Right Ventricular Pacing, CTR: Cardiac Resynchronization Therapy, TTP: The Time to Peak, ROI: Region of Interest, GLS: Global Longitudinal Peak Systolic Strain

\section{ABSTRACT}

Purpose: Single site ventricular pacing can result in electromechanical dyssynchrony and LV dysfunction. A classic pattern by strain echocardiography pattern analysis has been defined that specifically identifies electromechanical dyssynchrony that leads to $\mathrm{LV}$ dysfunction in other populations. Our purpose is to evaluate for the presence of the classic pattern and an association with LV dysfunction in asymptomatic young subjects with chronic pacing.

Methods: Inclusion criteria of non-surgical 2nd or 3rd degree AV block, normal cardiac anatomy, chronic ventricular pacing ( $>95 \%$ of beats) and a quality 4 -chamber apical image were met by 24 retrospective and 10 prospectively enrolled subjects. Global longitudinal peak strain (GLS), the classic pattern of dyssynchrony, and time to peak strain were analyzed with Tomtec 2D strain software. Statistics included Spearman's rank sum and Fisher's exact test.

Results: Median age at study echo was 18.4 years (9.8, 22.5 years); all had prolonged QRS duration with paced BBB pattern. The classic pattern was seen in $38 \%(13 / 34)$ and was associated with a lower LVEF (37.5 vs 48.9\%, $\mathrm{p}=0.001)$ and worse GLS (-10.7 vs $-14.8 \%, p=0.03)$. Pacing site was not associated with the classic pattern ( $p=0.7)$. Only in subjects with the classic pattern was pacing duration associated with progressive LV dysfunction.

Conclusion: LV dysfunction was common in this asymptomatic population suggesting routine echocardiographic surveillance is important. The classic pattern of electromechanical dyssynchrony was commonly identified in this ventricular paced population and was associated with more significant LV dysfunction. Larger future studies with prospective, serial echocardiograms are necessary to confirm these findings. 


\section{Introduction}

Young subjects with complete atrioventricular (AV) block eventually require life-long ventricular pacing. Although right ventricular pacing (RVP) provides the necessary therapy, recent studies have demonstrated adverse long-term effects on left ventricular (LV) function [1-5]. Right ventricular pacing frequently leads to electromechanical dyssynchrony due to the delayed activation of the LV lateral wall [6]. Electromechanical dyssynchrony progressively leads to pumping inefficiencies, maladaptive ventricular remodeling, systolic and diastolic dysfunction, and clinical heart failure [5,7-13]. Echocardiographic speckle tracking strain analysis allows for identification of mechanical dyssynchrony [12-13]. While there are many echocardiographic dyssynchrony indices, recently, regional strain pattern analysis has been used to more specifically identify a classic pattern of electromechanical dyssynchrony. This classic pattern has been identified in children [11] and adults [14] with native LBBB and in congenital heart disease patients $[12,13,15]$. This pattern is characterized by paradoxical wall motion resulting from early contraction of the septal wall versus late contraction in the activation-delayed lateral wall.

Cardiac resynchronization therapy (CRT) can treat electromechanical dyssynchrony when properly diagnosed and has been shown to reduce heart failure hospitalizations and deaths, improve functional status, and cause favorable ventricular reverse remodeling in the majority of patients $[16,17]$. While many echo dyssynchrony criteria, such as the time to peak (TTP) criteria, have not proven effective in improving CRT response rates as they evaluate for general mechanical discoordination rather than electromechanical dyssynchrony, [8] the classic pattern has sensitivity and specificity $>90 \%$ for predicting CRT response in adults with native LBBB [14]. This observational study is the first to identify the classic pattern in young subjects with chronic RVP and assess the relationship with LV dysfunction. Identification of pacing induced dyssynchrony is important in this $\mathrm{AV}$ block population due to their life-long pacing requirements and the fact that progressive dysfunction is often seen in patients with electromechanical dyssynchrony.

\section{Methods}

\section{Study Population}

We retrospectively identified subjects from the Duke Pediatric Pacemaker Database from 2005-2011. Inclusion criteria included:

a) All subjects with non-surgical second or third-degree AV block,

b) Normal cardiac anatomy; and

c) Chronic ventricular pacing of more than 95\% of cardiac cycles over entire duration of follow-up with a dual-chamber pacemaker. Subjects were excluded if they did not have at least one echocardiogram with an adequate LV 4 chamber apical view for strain analysis obtained after implantation of the pacing system. All retrospectively identified patients meeting inclusion criteria $(n=32)$ were evaluated for exclusion criteria. Eight patients were excluded due to inadequate imaging quality for strain analysis on qualifying echocardiograms. Twenty-four retrospectively identified subjects met all criteria. In addition, we prospectively enrolled ten subjects from the Duke Pediatric Pacemaker Clinic seen in 2012-2013 meeting all study criteria, for a total of 34 subjects (24 retrospective and 10 prospective). The prospectively enrolled subjects received a clinically indicated prospective echo optimized for strain analysis.

Determination of maternal anti-Ro or anti-La antibody status or other cardiomyopathy laboratory testing was not consistently performed and, therefore, was not included in this analysis. None of the included subjects had a known diagnosis of cardiomyopathy. This study was approved by the Duke University Medical Center Institutional Review Board, and all prospectively enrolled subjects provided informed consent.

\section{Electrocardiography}

A standard 12-lead ECG obtained nearest in time to the echocardiogram utilized for strain analysis in the retrospective cohort was identified. Prospectively enrolled subjects had an ECG obtained on the same day as the echo. The R-R and QRS intervals were measured. Due to the wide range of subject ages (3.1 - 39.5 years) and the expected changes in the QRS duration across that age range in this mixed pediatric and adult population, an adjusted QRS duration was calculated by dividing the QRS duration by the square root of the R-R interval to provide an indexed QRS duration (QRSd ${ }_{\text {adj }}$ ) [16]. Bundle branch block was determined based on standard criteria [18]. Pacemaker type and settings were recorded from pacemaker interrogation reports. Implantation dates of each pacemaker system and the percentage of ventricular paced beats as a percentage of total ventricular beats at least since the previous device interrogation were also reported. Pacing system duration defined as time from initial or current pacer insertion until date of echocardiogram.

\section{Echo Image Acquisition}

Retrospectively identified subjects had studies obtained on various vendors' ultrasound systems between 1/2007 and 3/2011. Assessment of serial echocardiograms was not feasible because an echo lab protocol leading to adequate acquisition quality for strain analysis was not consistently implemented until 2007. Prospective studies were obtained on a GE Vivid 7 (GE, Vingmed Ultrasound, Horten, Norway). The grayscale images were optimized for longitudinal speckle tracking strain analysis. All studies were exported to the Xcelera (Philips Medical Systems) server with standard data compression. All studies included a four-chamber apical view, LV outflow tract interrogation by pulsed wave spectral Doppler, and ECG tracing of sufficient quality for strain analysis. A LV ejection fraction (LVEF) was measured using the single plane Simpson's Rule [19]. 


\section{Velocity Vector Imaging Tomtec Strain Analysis}

All prospective and retrospective studies were converted to DICOM format and imported into Tomtec (Cardiac Performance Analysis Software, Siemens Medical Solutions, Mountain View, CA). Longitudinal 2D Velocity Vector Imaging strain analysis was performed on a single LV four-chamber image. The analyzed cardiac cycle was timed to the onset of the $Q$ wave to ensure that early systolic events were not missed. A single region of interest (ROI) was placed along the endocardial side of the myocardium. The integrity of the speckle-tracking was automatically detected and visually confirmed. In case of poor tracking, the tracing was readjusted, and segments with persistent inadequate tracking were excluded from analysis. The timing of aortic valve opening (AVO) and closing (AVC) were defined on the spectral Doppler tracing. Feasibility was determined as the percent of all segments with adequate tracking. Segmental peak systolic longitudinal strain was measured in six LV segments. Global longitudinal peak systolic strain (GLS) was calculated as an average of the six segmental strains. GLS is a negative percentage by convention. A larger negative GLS represents increased strain (eg, a patient with GLS of -15\% has greater ventricular contractility than one with -11\%). Longitudinal strain analysis on compressed Tomtec images demonstrate good reproducible and minimal bias compared with analysis on raw data images in pediatric and adult age groups [20,21].
Contraction timing was measured using segmental time to peak (TTP) intervals as the time from the onset of the QRS to the primary distinct peak on a strain curve. The standard deviation of the six segmental TTP values for each patient was calculated as an index of mechanical discoordination. A standard deviation of TTP (TTPsd) $\geq$ 60 milliseconds has been used as a traditional marker of significant dyssynchrony in adults with LBBB-associated cardiomyopathy [22].

\section{Regional Strain Pattern Analysis to Identify the Classic Pattern}

Methodology for regional strain pattern analysis has been previously published in multiple populations to identify a classic pattern consistent with electromechanical dyssynchrony based on longitudinal strain analysis. [14,23] The following three criteria define the classic pattern:

a) early terminated contraction of at least one septal segment simultaneous with early stretch in at least one opposing lateral wall segment

b) early terminated peak contraction in the septum must occur in the first $70 \%$ of the systolic ejection phase; and

c) at least one lateral wall segment demonstrates early stretch followed by late contraction (peaking after AVC) [14] (Figure 1).

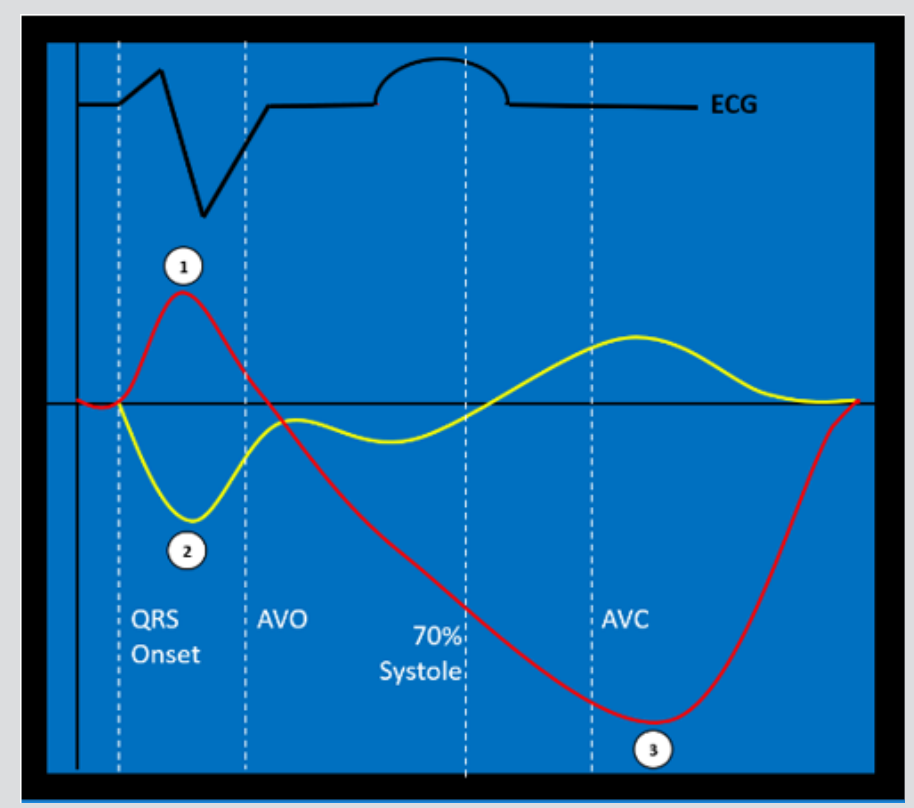

Figure 1: The classic pattern of electromechanical dyssynchrony. Numbers identify the three criteria to define the pattern on two representative strain curves (septum: yellow, lateral wall: red):

a) early contraction of at least one septal segment (yellow tracing) and early stretching in at least one opposing lateral wall segment (red tracing)

b) early peak contraction must occur in the first $70 \%$ of the systolic ejection phase (from aortic valve opening to closure) and c) late post-systolic peak contraction occurring after aortic valve closure (AVC) in the segment which had demonstrated early stretch. ECG tracing (black) provided for timing. Aortic valve opening (AVO).

\section{Cardiac Resynchronization Therapy}

Cardiac resynchronization therapy has only been performed in four of the study subjects. In these subjects, primary strain analy- sis was performed on an echocardiogram prior to CRT. Follow-up echocardiograms were obtained on all four CRT subjects between 4 months and 5.3 years after CRT. CRT response was defined as an improvement in LVEF > 10\%. 


\section{Statistical Analysis}

Continuous variables were reported as the median value $\left(25^{\text {th }}\right.$ $-75^{\text {th }}$ percentiles) unless otherwise specified and compared across groups using Wilcoxon rank sum and Kruskal-Wallis tests where appropriate. Dichotomous and categorical variables were reported as an absolute number (\%) and compared using Chi square or

\section{Results}

\section{Study Population Characteristics}

Table 1: Cohort pacing characteristics and LV function at time of study echocardiogram. Pacing system duration defined as time from pacer insertion to date of echocardiogram. Year (yr); left ventricular (LV); right ventricular (RV); QRS duration divided by the square root of the R-R interval $\left(\mathrm{QRSd}_{\mathrm{adj}}\right.$ ), bundle branch block (BBB); milliseconds (ms); left ventricular ejection fraction (LVEF); left ventricular global longitudinal peak systolic strain (LV GLS). Continuous variables were reported as median (25, $75 \%$ ile).

\begin{tabular}{|c|c|}
\hline Pacing \& Echo Characteristics & \\
\hline Total pacing duration (yr) & $8.0(5.6,13.1)$ \\
\hline Current pacing system duration (yr) & $4.7(2.0,7.3)$ \\
\hline \multicolumn{2}{|l|}{ Lifetime Pacing Systems } \\
\hline 1 & $10(29 \%)$ \\
\hline 2 & $16(47 \%)$ \\
\hline$\geq 3$ & $8(24 \%)$ \\
\hline \multicolumn{2}{|l|}{ Device Type at Time of Echo } \\
\hline RV Transvenous & $26(76 \%)$ \\
\hline RV Epicardial & $6(18 \%)$ \\
\hline LV Epicardial & $2(6 \%)$ \\
\hline \multicolumn{2}{|l|}{ Ventricular Lead Location } \\
\hline RV apical septum & $11(32 \%)$ \\
\hline RV mid septum & $15(44 \%)$ \\
\hline RV outflow tract & $6(18 \%)$ \\
\hline LV Apex & $2(6 \%)$ \\
\hline Paced QRSd (ms) & $138(128,148)$ \\
\hline Paced QRSd $d_{\text {adj }}(\mathrm{ms})$ & $150(136,168)$ \\
\hline BBB & $34(100 \%)$ \\
\hline $\operatorname{LVEF}(\%)$ & $45(37,51)$ \\
\hline LV GLS (\%) & $-13.3(-10.2,-16.2)$ \\
\hline
\end{tabular}

The age of this cohort at the time of the study echocardiogram was 18.6 years $(9.8,22.5$ years). Ten subjects were below the age of 10 years (range $3.1-9.6$ years). The median body surface area was $1.6 \mathrm{~m} 2$ (1.1 - $1.8 \mathrm{~m} 2)$, and 53\% were male. Thirty of the subjects had congenital AVB (88\%), and the other four (12\%) had early, non-surgical acquired AVB. No study subjects had reported any symptoms of heart failure (chest pain, shortness of breath, exercise intolerance, syncope, dyspnea on exertion). Pacing and ventricular function characteristics are described (Table 1). Both LVEF and LV GLS were, on average, mildly to moderately diminished in this population. There is strong correlation between GLS and LVEF ( $\mathrm{r}$ $=-0.82, \mathrm{p}<0.001)$. Most subjects $(23 / 34,68 \%)$ had LVEF $<50 \%$. Six subjects (17\%) demonstrated at least moderate LV dysfunction (LVEF $<40 \%$ and GLS $<-10 \%$ ).
Fisher's exact tests. Correlations between continuous variables were measured using Spearman's rank test. Logistic regression was used to model predictors of the classic pattern (defined as a dichotomous variable). A p-value $<0.05$ was considered statistically significant. Statistical analyses were performed using STATA 13.1 (College Station, TX). 
paced and $\mathrm{LV}$ ejection fraction $(\mathrm{r}=-0.68, \mathrm{p}=0.01)$, but in those without the classic pattern, there was no such association $(r=0.072$, $\mathrm{p}=0.76)$. Overall, the TTP $\mathrm{sd}_{\text {d }}$ ranged from normal to dyssynchronous (56ms, 38 - $129 \mathrm{~ms}$ ). Fourteen subjects (41\%) met the threshold for significant dyssynchrony by this $\mathrm{TTP}_{\text {sd }}$ criteria ( $>60$ milliseconds). The classic pattern and $\mathrm{TTP}_{\text {sd }}>60 \mathrm{~ms}$ criteria were strongly associated and the groups overlapped significantly, but there were three total subjects who met one but not both criteria (Table 2).

Table 2: Characteristics of those with and without the classic pattern of electromechanical dyssynchrony. Total duration of pacing (Total duration); duration of current pacing system (Current duration); duration of the paced QRS indexed to the square root R-R duration (QRS ${ }_{\text {adi }}$ duration); year (yr); left ventricular (LV); right ventricular (RV); milliseconds (ms); left ventricular ejection fraction (LVEF); left ventricular global longitudinal peak systolic strain (LV GLS); standard deviation of the six segmental time to peak contraction $\left(\mathrm{TTP}_{\mathrm{sd}}\right)$. Continuous variables were reported as median $(25,75 \%$ ile $)$.

\begin{tabular}{|c|c|c|c|}
\hline & Classic & None & $\mathbf{P}$ \\
\hline Subjects & $13(38 \%)$ & $21(62 \%)$ & \\
\hline Total paced duration (yr) & $8.7(5.6,14.0)$ & $7.7(5.1,11.1)$ & 0.61 \\
\hline Current pacing system duration (yr) & $4.0(1.1,7.2)$ & $4.7(3.1,7.3)$ & 0.63 \\
\hline $\mathrm{QRSd}_{\mathrm{ad}} \mathrm{j}(\mathrm{ms})$ & $163(148,172)$ & $142(127,158)$ & 0.04 \\
\hline $\operatorname{LVEF}(\%)$ & $37.5(28.9,42.1)$ & $48.9(40.8,53.5)$ & 0.001 \\
\hline LV GLS (\%) & $-10.7(-8.2,-14.9)$ & $-14.8(-11.4,-16.4)$ & 0.03 \\
\hline Pacing Site & & & 0.76 \\
\hline RV Apex & $3(12 \%)$ & $8(20 \%)$ & \\
\hline RV Septum & $7(21 \%)$ & $8(23 \%)$ & \\
\hline $\mathrm{RV}$ outflow tract & $2(6 \%)$ & $4(12 \%)$ & \\
\hline LV Apex & $1(3 \%)$ & $1(3 \%)$ & \\
\hline Time to Peak (TTP) & & & $<0.001$ \\
\hline $\mathrm{TTP}_{\mathrm{sd}} \geq 60 \mathrm{~ms}$ & $12(35 \%)$ & $2(6 \%)$ & \\
\hline $\mathrm{TTP}_{\mathrm{sd}}<60 \mathrm{~ms}$ & $1(3 \%)$ & $19(56 \%)$ & \\
\hline
\end{tabular}

\section{Speckle Tracking Feasibility}

Adequate tracking by the strain analysis software was achieved in $90 \%$ of all segments (183/204 adequate), demonstrating good feasibility in the study subjects. The apical lateral wall segment was the least well tracked at $71 \%$ feasibility (24/34 adequate).

\section{Cardiac Resynchronization Therapy}

In the four subjects (ages 6, 12, 26, and 27 years) who underwent cardiac resynchronization therapy, the single ventricular lead pacing system was upgraded to biventricular pacing with either a transvenous coronary sinus lead in the three older subjects or an epicardial LV lead in the younger subject. All met criteria for both the classic pattern and TTP criteria pre-CRT with moderate or severely diminished LV function (LVEF: 29\%, 34\%, 40\%, $42 \%$ ). Follow-up ranged from 4 months to 5.3 years. All subjects demonstrated response with improvement in LVEF by $>10 \%$. The response in one subject's pre-CRT and post-CRT strain curves is illustrated in Figure 2.

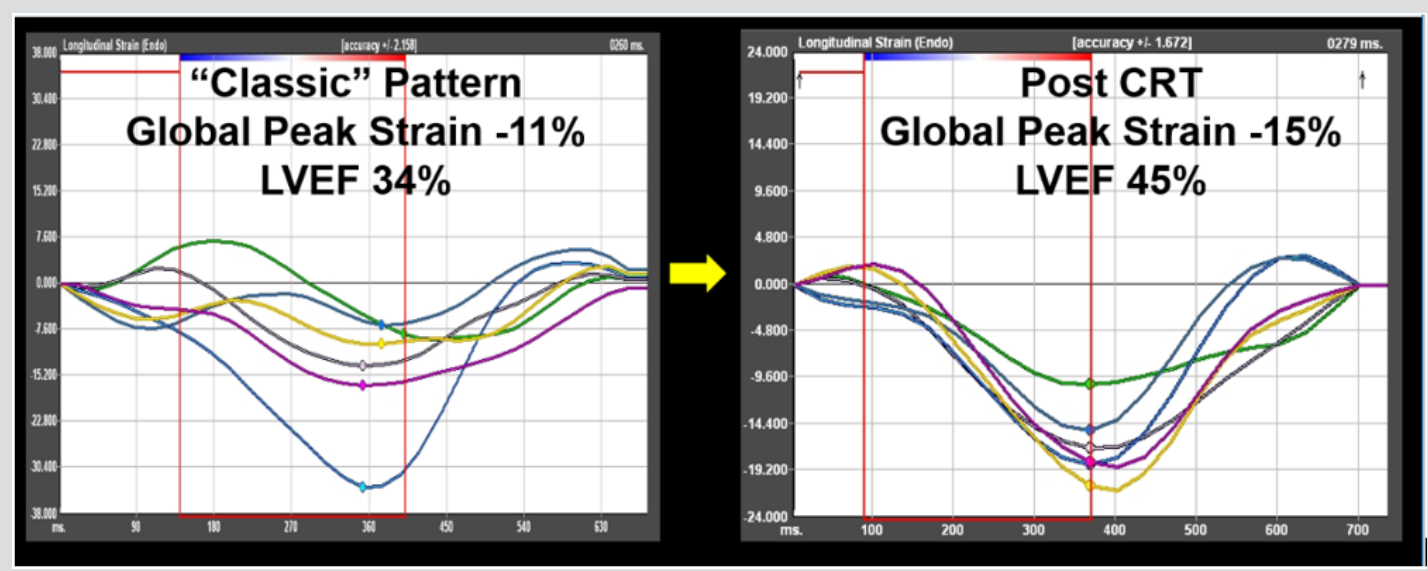

Figure 2: In this patient with a left bundle branch block-type pattern from right ventricular pacing, the pacing system was upgraded to biventricular pacing with a transvenous coronary sinus lead. The pre-CRT strain curves (left) demonstrate classic pattern with early terminated contraction seen in the basal and mid septum (yellow and dark blue) opposed by early stretch and late contraction in the basal lateral wall (green). He had severely diminished LV function. Follow-up echocardiogram at 4 months demonstrated significant improvement in LVEF, with a re-synchronization of the strain curves. 


\section{Discussion}

The main findings of this study of young asymptomatic patients with CAVB and single site ventricular pacing are:

a) there is an overall high frequency of clinically silent LV systolic dysfunction;

b) subjects with the classic pattern of electromechanical dyssynchrony had a lower LVEF and global longitudinal strain than those not having the classic pattern; and

c) Among those with a classic pattern, but not those without the pattern, the degree of LV dysfunction was associated with the duration of pacing.

\section{Chronic Pacing and LV Dysfunction}

Although chronic ventricular pacing is known to be associated with $\operatorname{LV}$ dysfunction $[5,6,10]$, we observed that the majority of our subjects fell into the abnormal range and that $17 \%$ of our population had at least moderate LV systolic dysfunction. None of the subjects having LV dysfunction, even those with moderatesevere dysfunction, reported any symptoms of heart failure, potentially due to the gradual nature of this process. In a large multicenter study of pediatric AV block subjects, Janousek et al. [5] found that $>50 \%$ of those with chronic RV pacing had diminished
LVEF $<55 \%$. Our observations in this asymptomatic cohort agree with previous literature and serve to emphasize the importance of routine echocardiographic surveillance in those with chronic ventricular pacing.

\section{Classic Pattern of Electromechanical Dyssynchrony}

This observational study demonstrates that identification of the classic pattern using strain analysis was associated with more significant cardiac dysfunction. In adults with native LBBB, the classic pattern criteria were based on longitudinal strain and designed to represent the consequences of a pathophysiological sequence in which electrical activation delays lead to electromechanical dyssynchrony, systolic dysfunction, and eventually heart failure (Figure 3) [14]. The classic pattern, found in subgroups of various populations with electromechanical dyssynchrony [11, 14, 15, 2327], was also identified in $38 \%$ of the current ventricular paced young patient cohort having normal cardiac anatomy. In subjects who require lifelong RVP, the classic pattern may help to identify a subgroup at increased risk for more severe dysfunction who may benefit from more frequent echo surveillance. Identification of the classic pattern may also more specifically identify those who will respond to an upgraded biventricular pacing system, but that was not systematically evaluated in this study.

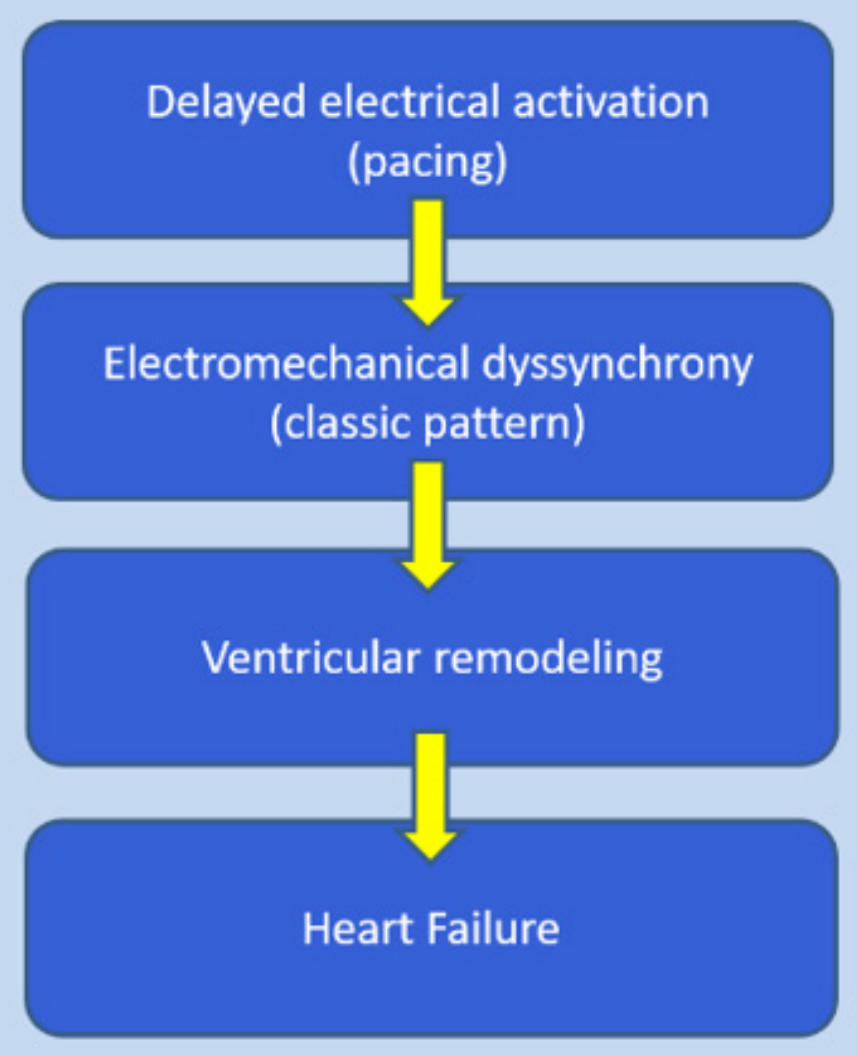

Figure 3: The pathophysiologic sequence of electrical activation leading to dyssynchrony, ventricular remodeling and eventually heart failure. 


\section{Non-Classic Pattern Population}

In our study population, those without the classic pattern (62\%) had significantly better LV function, but some had low-normal or mildly diminished systolic function. Nearly all of the subjects who did not have the classic pattern, but did have mild LV dysfunction, had congenital AV block. Previous studies have shown that primary myocyte dropout and fibrosis secondary to in utero immunemediated mechanisms from maternally acquired autoimmune antibodies may contribute to fetal and infantile cardiomyopathy [20-21]. Such a mechanism could result in a regionally discoordinated LV strain pattern (positive for TTP criteria) that would not meet classic pattern criteria as it does not arise from electromechanical dyssynchrony. This may explain the two subjects who were positive for the $\mathrm{TTP}_{\text {sd }}$ criteria but negative for the classic pattern. Additionally, this pro-fibrotic mechanism may account for LV ventricular dysfunction through a different mechanism than electromechanical dyssynchrony in this population.

\section{Duration of Pacing and Ventricular Dysfunction}

A recent multi-center study demonstrated that total duration of pacing did not predict LV dysfunction [4]. In our study, the total duration of pacing was similar between subjects with and without a classic pattern. The development of the classic pattern is not dependent on longer pacing duration. However, in those with the classic pattern, deterioration in LVEF is dependent on the pacing duration. Those without the classic pattern did not have a timedependent relationship with LV dysfunction. This would suggest that development of significant electromechanical dyssynchrony identified by the classic pattern leads to progressive pacing induced dysfunction over time. Clinically, this information may potentially allow for triaging of paced patients into those with the classic pattern requiring more frequent echo monitoring and those without the pattern not requiring as frequent monitoring.

\section{Site of Pacing and Abnormal LV Mechanics}

The location of a single ventricular pacing lead has been shown to be associated with increased dysfunction, although the location associated with more severe dysfunction has varied by study $[5,7,10]$. A recent multi-center study that considered lead location and LV dysfunction demonstrated that the RVOT leads were associated with the poorest LV function, although there were only 9 subjects in this subgroup [5]. All RV lead locations were associated with diminished LV function compared to LV epicardial locations. There was no association between RV pacing site and the development of the classic pattern in our study. This may suggest that the abnormal activation from single site pacing with any RV lead location may cause this pattern of electromechanical dyssynchrony given the right circumstances. However, this analysis is underpowered to answer this question, and any other necessary circumstances required for the development of the classic pattern are unknown. Left ventricular pacing from the apex or lateral wall has been shown to better maintain normal LV function in multiple studies $[5,28]$. In our study, there were only two subjects with epicardial LV pacing. One had normal function and no dyssynchrony. The other had mildly diminished function and a classic pattern with early apical and late basal contraction. This type of classic pattern with early LV apical contraction and late basal contraction is similar to the classic pattern seen with pacing from the apical RV septum [6]. The interplay between the type of pacer, the pacing lead site and the development of electromechanical dyssynchrony and progressive dysfunction will require future research.

\section{CRT Response}

Only four of our study subjects had undergone CRT with placement of a coronary sinus or LV epicardial lead at the time of this study. All four subjects had the classic pattern and met TTP criteria prior to undergoing CRT. They all demonstrated significant beneficial LV reverse remodeling post-CRT. None of these subjects ever reported symptoms of heart failure before or after CRT. Biventricular CRT is known to improve ventricular function compared to RV pacing in the AV block population [29]. However, it is unknown whether the classic pattern can improve CRT response rates over EKG only or EKG and TTP criteria in this paced population as it has in native LBBB cardiomyopathy [14, 23]. This study was not designed to evaluate the classic pattern or TTP indices to predict CRT response in this AVB population.

\section{Limitations}

The echo lab standard changed in 2007 when 4 chamber apical views became consistently of adequate quality for strain analysis. Because only one echocardiographic evaluation of adequate quality was performed for many of our subjects, this study could not determine when the classic pattern or ventricular dysfunction first developed. An effort to analyze echocardiograms immediately after pacemaker implantation was made impossible due to the variation in echo image quality in many of those studies that were performed before the current echo lab standard was in place and using ultrasound machines and imaging standards from a previous era. Furthermore, the LV3 and LV2 apical and parasternal short axis views were not consistently obtained at adequate quality for strain analysis during the study period, so evaluation of the 18 segment GLS or other strain planes were not feasible. The lack of LV3 and LV2 apical views may have led to a slight under-identification of the classic pattern, but Risum et al. [14] demonstrated that for nearly all subjects with the classic pattern, it is present in the LV four chamber view. Our study provides early data identifying a previously unrecognized association between the classic pattern and progressive dysfunction in this population that will require future prospective study. This study is also not designed to evaluate CRT response in subjects with the classic pattern. Due to incomplete records, the maternal immunologic status (anti-Ro/La) in our study population was not available. 


\section{Conclusion}

This study demonstrates a high frequency of clinically silent but significant LV dysfunction in young subjects having CAVB who require chronic single site ventricular pacing. In subjects with a classic pattern of electromechanical dyssynchrony by strain analysis, a strong correlation was found between duration of pacing and LV dysfunction that was not present in those without the classic pattern. The classic pattern identifies a group of RV paced subjects who may require closer echo follow-up. The findings from our study should lead to a larger study with serial echocardiograms prospectively protocoled for strain analysis to more thoroughly evaluate the development and consequences of the classic pattern in this young paced population.

\section{References}

1. Karpawich PP, Rabah R, Haas JE (1999) Altered cardiac histology following apical right ventricular pacing in patients with congenital atrioventricular block. Pacing Clin Electrophysiol 22(9): 1372-1377.

2. Moak JP, Hasbani K, Ramwell C, Freedenberg V, Berger JT, et al. (2006) Dilated cardiomyopathy following right ventricular pacing for AV block in young patients: resolution after upgrading to biventricular pacing systems. J Cardiovasc Electrophysiol 17(10): 1068-1071.

3. Kim JJ, Friedman RA, Eidem BW, Cannon BC, Arora G, et al. (2007) Ventricular function and long-term pacing in children with congenital complete atrioventricular block. J Cardiovasc Electrophysiol 18(4): 373377.

4. Gebauer RA, Tomek V, Salameh A, Marek J, Chaloupecký V, et al. (2009) Predictors of left ventricular remodelling and failure in right ventricular pacing in the young. Eur Heart J 30(9): 1097-1104.

5. Janousek J, van Geldorp IE, Krupickova S, Rosenthal E, Nugent K, et al (2013) Permanent cardiac pacing in children: choosing the optimal pacing site: a multicenter study. Circulation 127(5): 613-623.

6. Tanaka H, Hara H, Adelstein EC, Schwartzman D, Saba S, et al. (2010) Comparative mechanical activation mapping of RV pacing to LBBB by $2 \mathrm{D}$ and $3 \mathrm{D}$ speckle tracking and association with response to resynchronization therapy. JACC Cardiovasc Imaging 3(5): 461-471.

7. Thambo JB, Bordachar P, Garrigue S, Lafitte S, Sanders P, et al. (2004) Detrimental ventricular remodeling in patients with congenital complete heart block and chronic right ventricular apical pacing. Circulation 110(25): 3766-3772.

8. Tops LF, Suffoletto MS, Bleeker GB, Boersma E, van der Wall EE et al. (2007) Speckle-tracking radial strain reveals left ventricular dyssynchrony in patients with permanent right ventricular pacing. J Am Coll Cardiol 50(12): 1180-1188.

9. Salameh A, Dhein S, Blanke K, Rastan A, Hiyasat B, et al. (2012) Right or left ventricular pacing in young minipigs with chronic atrioventricular block: long-term in vivo cardiac performance, morphology, electrophysiology, and cellular biology. Circulation 125(21): 2578-2587.

10. Ahmed M, Gorcsan J, Marek J, Ryo K, Haugaa K, et al. (2014) Right ventricular apical pacing-induced left ventricular dyssynchrony is associated with a subsequent decline in ejection fraction. Heart rhythm 11(4): 602-608.

11. Forsha D, Slorach C, Chen CK, Stephenson EA, Risum N, et al. (2014) Classic-pattern dyssynchrony and electrical activation delays in pediatric dilated cardiomyopathy. J Am Soc Echocardiogr 27(9): 956-964.

12. Forsha D, Risum N, Barker P (2017) Activation delay-induced mechanical dyssynchrony in single-ventricle heart disease. Cardiol Young 27(7): 1390-1391.
13. Forsha D, Risum N, Smith PB, Kanter RJ, Samad Z, et al. (2016) Frequent Activation Delay-Induced Mechanical Dyssynchrony and Dysfunction in the Systemic Right Ventricle. J Am Soc Echocardiogr 29(11): 1074-1083.

14. Risum N, Jons C, Olsen NT, Fritz-Hansen T, Bruun NE, et al. (2012) Simple regional strain pattern analysis to predict response to cardiac resynchronization therapy: rationale, initial results, and advantages. Am Heart J 163(4): 697-704.

15. Forsha D, Risum N, Kropf PA, Rajagopal S, Smith PB, et al. (2014) Right Ventricular Mechanics Using a Novel Comprehensive Three-View Echocardiographic Strain Analysis in a Normal Population. J Am Soc Echocardiogr 27(4): 413-422.

16. Epstein AE, DiMarco JP, Ellenbogen KA, Estes NA $3^{\text {rd }}$, Freedman RA, et al. (2008) ACC/AHA/HRS 2008 Guidelines for Device-Based Therapy of Cardiac Rhythm Abnormalities: a report of the American College of Cardiology/American Heart Association Task Force on Practice Guidelines (Writing Committee to Revise the ACC/AHA/NASPE 2002 Guideline Update for Implantation of Cardiac Pacemakers and Antiarrhythmia Devices): developed in collaboration with the American Association for Thoracic Surgery and Society of Thoracic Surgeons. Circulation 117(21): e350-408.

17. Cleland JG, Daubert JC, Erdmann E, Nick Freemantle, Daniel Gras, et al. (2005) The effect of cardiac resynchronization on morbidity and mortality in heart failure. New Engl J Med 352: 1539-1549.

18. Surawicz B, Childers R, Deal BJ, Gettes LS, Bailey JJ, et al. (2009) AHA/ ACCF/HRS recommendations for the standardization and interpretation of the electrocardiogram: part III: intraventricular conduction disturbances: a scientific statement from the American Heart Association Electrocardiography and Arrhythmias Committee, Council on Clinical Cardiology; the American College of Cardiology Foundation; and the Heart Rhythm Society. Endorsed by the International Society for Computerized Electrocardiology. J Am Coll Cardiol 53(11): 976-981.

19. Lang RM, Bierig M, Devereux RB, Flachskampf FA, Foster E, et al. (2005) Recommendations for chamber quantification: a report from the American Society of Echocardiography's Guidelines and Standards Committee and the Chamber Quantification Writing Group, developed in conjunction with the European Association of Echocardiography, a branch of the European Society of Cardiology. J Am Soc Echocardiogr 18(12): 1440-1463.

20. Koopman LP, Slorach C, Manlhiot C, Manlhiot C, McCrindle BW,et al. (2011) Assessment of myocardial deformation in children using Digital Imaging and Communications in Medicine (DICOM) data and vendor independent speckle tracking software. J Am Soc Echocardiogr 24(1): $37-44$.

21. Risum N, Ali S, Olsen NT, Jons C, Khouri MG, et al. (2012) Variability of global left ventricular deformation analysis using vendor dependent and independent two-dimensional speckle-tracking software in adults. J Am Soc Echocardiogr 25(11): 1195-1203.

22. Mor Avi V, Lang RM, Badano LP, Belohlavek M, Cardim NM, et al. (2011) Current and evolving echocardiographic techniques for the quantitative evaluation of cardiac mechanics: ASE/EAE consensus statement on methodology and indications endorsed by the Japanese Society of Echocardiography. J Am Soc Echocardiogr 24(3): 277-313.

23. Risum N, Tayal B, Hansen TF, Bruun NE4, Jensen MT, et al. (2015) Identification of Typical Left Bundle Branch Block Contraction by Strain Echocardiography Is Additive to Electrocardiography in Prediction of Long-Term Outcome After Cardiac Resynchronization Therapy. J Am Coll Cardiol 66(6): 631-641.

24. Hui W, Slorach C, Dragulescu A, Mertens L, Bijnens B, et al. (2014) Mechanisms of right ventricular electromechanical dyssynchrony and mechanical inefficiency in children after repair of tetralogy of fallot. Circ Cardiovasc Imaging 7(4): 610-618.

25. Rosner A, Khalapyan T, Dalen H, McElhinney DB, Friedberg MK, et al. (2018) Classic-Pattern Dyssynchrony in Adolescents and Adults with a Fontan Circulation. J Am Soc Echocardiogr 31(2): 211-219. 
26. Forsha D, Risum N, Barker P (2017) Activation delay-induced mechanical dyssynchrony in single-ventricle heart disease. Cardiol Young 27(7): 1390-1391.

27. Klein MR, Sundh F, Simlund J, Harrison JK, Jackson KP, et al. (2015) Immediate mechanical effects of acute left bundle branch block by speckle tracked strain. J Electrocardiol 48(4): 643-651.

28. Tomaske M, Breithardt OA, Bauersfeld U (2009) Preserved cardiac synchrony and function with single site left ventricular epicardial pacing

ISSN: 2574-1241

DOI: 10.26717/BJSTR.2019.20.003435

Daniel Forsha. Biomed J Sci \& Tech Res

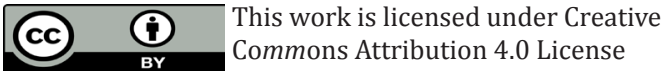

Submission Link: https://biomedres.us/submit-manuscript.php during mid-term follow-up in paediatric patients. Europace 11(9): 1168-1176.

29. St John Sutton M, Plappert T, Adamson PB, Li P, Christman SA, et al (2015) Left Ventricular Reverse Remodeling with Biventricular Versus Right Ventricular Pacing in Patients with Atrioventricular Block and Heart Failure in the BLOCK HF Trial. Circ Heart Fail 8(3): 510-518.

$\begin{array}{ll}\text { BIOMEDICAL } & \text { Assets of Publishing with us } \\ \text { - GlobeARCHES } & \text { - Immediate, unrestricted online access } \\ & \text { - Rigorous Peer Review Process } \\ & \text { - Authors Retain Copyrights }\end{array}$

